\title{
A Case of Eosinophilic Pleural Effusion Caused by Toxocariasis without Serum Eosinophilia
}

\author{
Min Ji Cho, Ju Ock Kim, Sung Soo Jung, Hee Sun Park, Da Hyun Kang, Dong II Park \\ Division of Pulmonology, Department of Internal Medicine, Chungnam National University School of Medicine, Daejeon, Korea
}

\begin{abstract}
Human toxocariasis is a parasitic disease caused by Toxocara canis or Toxocara cati. We report a patient with toxocariasis who presented with dyspnea and left pleural effusion. All patients with toxocariasis in published case reports had serum eosinophilia. This is the first case report of eosinophilic pleural effusion without serum eosinophilia caused by toxocariasis infection, which was confirmed by using enzyme-linked immunosorbent assay (ELISA). Therefore, an antibody ELISA can be useful for diagnosing toxocariasis in patients with a pleural effusion of unknown cause, even in the absence of serum eosinophilia.
\end{abstract}

Keywords: Toxocariasis; Pleural effusion; Eosinophilia

\section{INTRODUCTION}

Human toxocariasis is a parasitic disease caused by infection with larvae of Toxocara canis or Toxocara cati. Humans can become accidental hosts of Toxocara species by ingesting embryonated eggs or live larvae. After ingestion, the second-stage larvae hatch in the small intestine and then penetrate the intestinal wall and migrate to other organs; this is known as visceral larva migrans [1].

Migration of larvae can cause eosinophilic inflammation in various organs, including pulmonary infiltration, and pleural effusion due to host allergic and inflammatory responses. Pulmonary involvement of toxocariasis can present as symptoms of cough, dyspnea, wheezing, chest discomfort, and eosinophilic pleural effusion, and it is usually accompanied by serum eosinophilia [2,3]. We report a patient with toxocariasis who presented with an eosinophilic pleural effusion without serum eosinophilia.

\section{CASE REPORT}

An 85-year-old man with complaints of modified Medical Research Council dyspnea scale grade 2 dyspnea and pleuritic chest pain, which was worsened by deep breathing and coughing, was admitted to the outpatient clinic in Chungnam National University Hospital. Chest radiography revealed a left pleural effusion that shifted in the decubitus view (Fig. 1), therefore, he was hospitalized.

He have been taking amlodipine $5 \mathrm{mg}$ once daily for hypertension for the previous 8 years. He had no history of tuberculosis. He have never had a pet, but have occasionally eaten raw meat about once a week. Physical examination did not reveal wheezing or crackles, but decreased breath sounds were noted in the left lower lung. His vital signs were within the normal ranges.

The laboratory results were as follows: serum white blood cell (WBC) count 7,970/ $\mathrm{mm}^{3}$ with no eosinophilia (segmented neutrophils $69 \%$; lymphocytes $22.3 \%$; monocytes $6.0 \%$; and eosinophils 1.5\%); lactate dehydrogenase (LDH) $411 \mathrm{IU} / \mathrm{L}$; C-reactive protein $0.5 \mathrm{mg} / \mathrm{dL}$; total protein $6.3 \mathrm{~g} / \mathrm{dL}$; and albumin $3.5 \mathrm{~g} / \mathrm{dL}$.

Thoracentesis was performed to determine the cause of the pleural effusion and to improve his symptoms. The pleural effusion study results were as follows: $\mathrm{pH} 7.4$; red blood cell count $2,760 / \mathrm{mm}^{3}$; WBC count 2,420/ $/ \mathrm{mm}^{3}$; total protein $4.3 \mathrm{~g} / \mathrm{dL}$; LDH $237 \mathrm{IU} / \mathrm{L}$; adenosine deaminase $21.2 \mathrm{IU} / \mathrm{L}$; and carcinoembryonic antigen 2.06 $\mathrm{ng} / \mathrm{mL}$. These results indicate that the pleural effusion was an exudate, based on Light's criteria. At that time, however, cytospin examination was not performed to determine the WBC distribution. 
Cho MJ, et al. - Pleural Effusion from Toxocariasis with No Serum Eosinophila

The cytological test result showed many lymphocytes but was negative for eosinophils and malignant cells. Blood cultures and pleural fluid Gram stain and culture also yielded negative results.

After thoracentesis, the symptoms and chest radiographic findings were improved, but we could not know the cause of the pleural effusion because no specific parenchymal lesion was detected on chest computed tomography (Fig. 2) and no specific finding was obtained from the effusion study. For these reasons, routine follow-up was planned. However, the patient did not attend the clinic on the appointed day.

Four months later, the patient was readmitted with the same symptoms, that is, dyspnea and pleuritic chest pain. Although the physical and laboratory examination results did not differ from the previous results, chest radiography revealed progression of left loculated effusion (Fig. 3B). A pleural biopsy and pleural fluid examination were performed. The biopsy revealed chronic active inflammation with no specific findings. For further evaluation, we recommended hospitalization, but the patient and his guardian
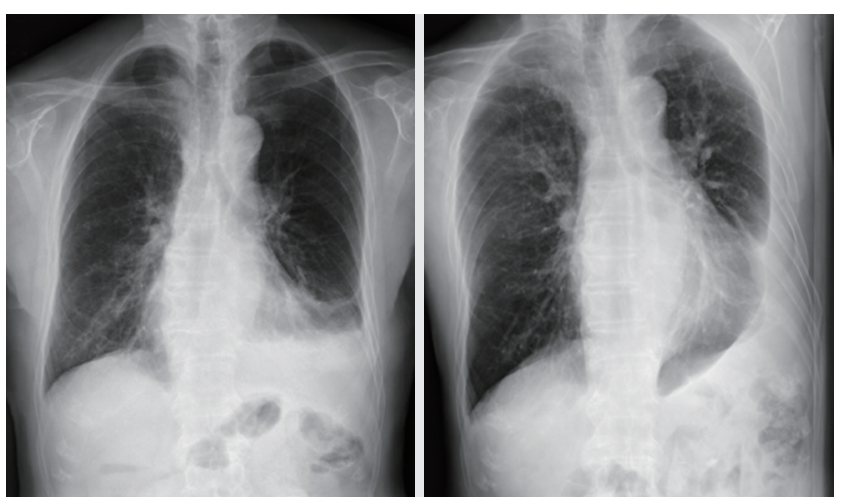

Fig. 1. Initial chest posteroanterior and decubitus view radiographs showing a left pleural effusion that shifted with the patient's position. refused because of his old age and the improvement in his symptoms after thoracentesis.

At that time, the pleural effusion results also showed exudate, but we missed the results of the pleural fluid examination, which revealed an eosinophilic effusion (eosinophils 35\%; lymphocytes $55 \%$; neutrophils 5\%; histiocytes $3 \%$; and mesothelial cells $2 \%$ ). Subsequently, the patient revisited the outpatient department when his symptoms worsened and underwent repeat thoracentesis. Over time, the rate of pleural effusion accumulation accelerated, so that the loculation worsened. Starting at 6 months after his first visit, the patient attended the clinic more than once per month.

No serum eosinophilia was found throughout the follow-up period. One year after the first visit, his pleural effusion worsened, so we conducted a chart review (Fig. 3C). This time, the missed eosinophilic pleural effusion was found, and then we could estimate

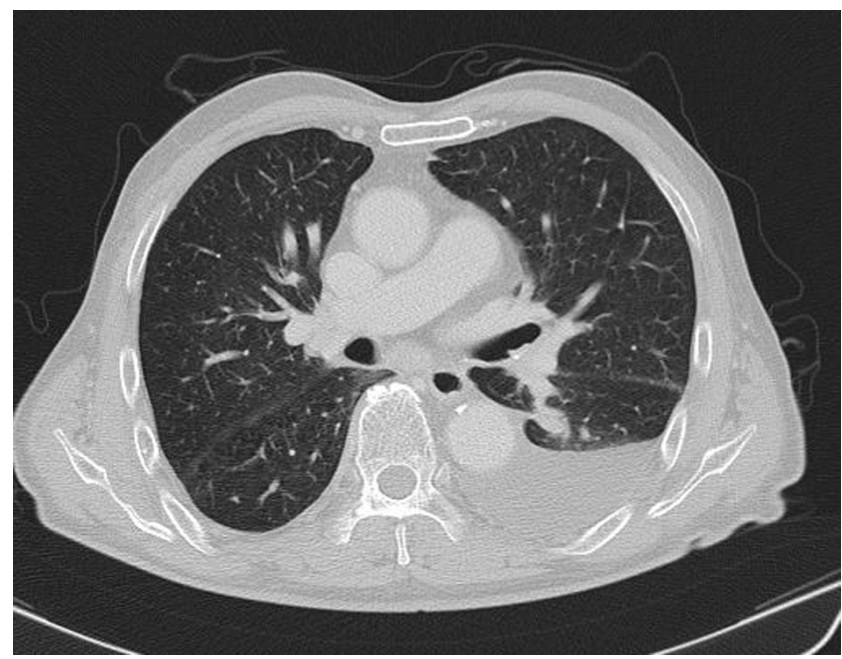

Fig. 2. Chest computed tomography scan showing left pleural effusion but no specific parenchymal lesion.
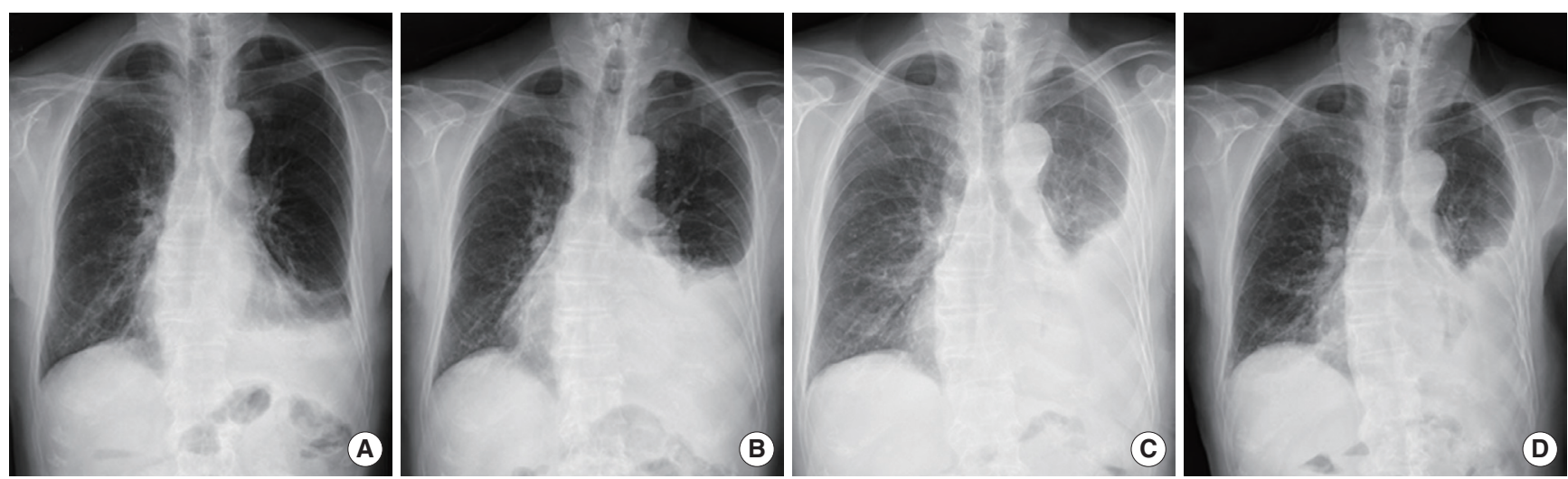

Fig. 3. Sequence of chest radiographs during treatment. (A) Initial chest radiograph in the posteroanterior view. (B) Chest radiographs 4 months after the initial presentation. (C) Chest radiograph obtained 1 year after and then albendazole was administered. (D) Chest radiograph obtained 6 months after oral administration of albendazole. 
his disease as a parasitic infection.

Serum toxocariasis antibody test results, performed using an enzyme-linked immunosorbent assay (ELISA), were positive at a titer of 1.181. The hospital ELISA test cut-off value was defined as a titer higher than 0.923 . Other parasite antibody tests, including clonorchiasis, cysticercus, sparganosis, and paragonimiasis, all yielded negative results (clonorchiasis immunoglobulin $\mathrm{G}[\operatorname{IgG}]$ 0.20; cysticercus IgG 0.18; sparganosis IgG 0.18; and paragonimiasis IgG 0.17). According to these results, toxocariasis was diagnosed.

The patient was prescribed albendazole ( $400 \mathrm{mg}$ twice daily for 5 days). He was not administered systemic corticosteroids. Upon examination at the follow-up visit 6 months after albendazole administration, we found that the loculated pleural effusion have not progressed (Fig. 3D).

\section{DISCUSSION}

Toxocara species induce eosinophilic inflammation due to host immune response, which can result in organ damage. The incidence rate of serum eosinophilia in toxocariasis is approximately $70 \%$ to $80 \%$ [3].

Hur et al. [4] identified 38 patients diagnosed with toxocariasis, of whom two had pleural effusion and one had no serum eosinophilia. Park et al. [5] identified 88 toxocariasis patients. In these patients, two had pleural effusion, one of whom had no serum eosinophilia [5]. However, these retrospective studies analyzed patients with positive toxocariasis antibody titers, which does not always indicate acute infection state, so that the cause-and-effect relationship is unclear. In fact, one study described the serum antibody positive rate of human toxocariasis in ELISA as $8.7 \%$ in healthy subjects in South Korea [6].

By contrast, all toxocariasis patients in published case reports had serum eosinophilia $[7,8]$. In other case reports, pulmonary involvement, but not pleural invasion, was accompanied by serum eosinophilia; the patients recovered after albendazole administration $[8,9]$.

Eosinophils degranulate onto parasites, kill them in the presence of antibodies, then move from the blood, and aggregate in the locality of the parasites. This explains why serum eosinophilia was not found during the evaluation in our case. In the acute phase, serum eosinophilia could be found, but at the time of his hospital admission for pleural effusion, eosinophils could be cleared from the blood and accumulate in the site of the thoracic cavity where the parasite antigen was present. Toxocariasis is generally diagnosed by using ELISA with anti-toxocariasis IgG. This test has 92.2\% sensitivity and $86.6 \%$ specificity. Biopsy provides a definitive diagnosis but is rarely performed because it is difficult to identify Toxocara larvae in tissue [10].

A 5-day-course of oral albendazole or mebendazole is recommended in the treatment of toxocariasis. In our case, the administration of albendazole inhibited progression of the loculated effusion but did not reduce it. Presumably, the pleural layer was already adherent and required more than antiparasitic therapy to resolve. Decortication was not performed considering the patient's advanced age and lack of symptoms.

This is the first case report of an ELISA-confirmed eosinophilic pleural effusion without serum eosinophilia caused by toxocariasis infection. Therefore, an antibody ELISA can be useful for diagnosing toxocariasis in patients with a pleural effusion of unknown cause but with risk factors such as consumption of raw meat or history of animal contact, even in the absence of serum eosinophilia.

\section{REFERENCES}

1. Lim JH. Toxocariasis of the liver: visceral larva migrans. Abdom Imaging 2008;33:151-6.

2. Huntley CC, Costas MC, Lyerly A. Visceral larva migrans syndrome: clinical characteristics and immunologic studies in 51 patients. Pediatrics 1965;36:523-36.

3. Kwon NH, Oh MJ, Lee SP, Lee BJ, Choi DC. The prevalence and diagnostic value of toxocariasis in unknown eosinophilia. Ann Hematol 2006;85: 233-8.

4. Hur JH, Lee IJ, Kim JH, Kim DG, Hwang HJ, Koh SH, et al. Chest CT findings of toxocariasis: correlation with laboratory results. Clin Radiol 2014;69:e285-90.

5. Park BM, Jeong SO, Park HS, Jung SS, Kim SY, Kim JO, et al. Differences in the clinical and radiological characteristics of lung-involved toxocariasis between toxocariasis with eosinophilia and those without eosinophilia. J Thorac Dis 2014;6:1757-64.

6. Kim HS, Jin Y, Choi MH, Kim JH, Lee YH, Yoon CH, et al. Significance of serum antibody test for toxocariasis in healthy healthcare examinees with eosinophilia in Seoul and Gyeongsangnam-do, Korea. J Korean Med Sci 2014;29:1618-25.

7. Jeanfaivre T, Cimon B, Tolstuchow N, de Gentile L, Chabasse D, Tuchais E. Pleural effusion and toxocariasis. Thorax 1996;51:106-7.

8. Yoshikawa M, Koyama N, Hontsu S, Yamamoto Y, Mikasa K, Kimura H. Lessons from eight cases of adult pulmonary toxocariasis: abridged republication. Respirology 2011;16:1014-5.

9. Ranasuriya G, Mian A, Boujaoude Z, Tsigrelis C. Pulmonary toxocariasis: a case report and literature review. Infection 2014;42:575-8.

10. Jin Y, Shen C, Huh S, Sohn WM, Choi MH, Hong ST. Serodiagnosis of toxocariasis by ELISA using crude antigen of Toxocara canis larvae. Korean J Parasitol 2013;51:433-9. 